\title{
Students' Beliefs about Willingness to Access Complementary and Alternative Therapies (CAT) Training for Future Integration into Psychology Practice
}

\author{
Lee-Ann M. Wilson, ${ }^{1}$ Kyra Hamilton, ${ }^{2,3}$ and Katherine M. White ${ }^{3}$ \\ ${ }^{1}$ School of Public Health, Queensland University of Technology, Victoria Park Road Kelvin Grove, Kelvin Grove, QLD 4059, Australia \\ ${ }^{2}$ School of Applied Psychology, Griffith University, Messines Ridge Road, MT Gravatt, QLD 4122, Australia \\ ${ }^{3}$ School of Psychology and Counselling, Queensland University of Technology, Victoria Park Road Kelvin Grove, Kelvin Grove, \\ QLD 4059, Australia
}

Correspondence should be addressed to Kyra Hamilton, k2.hamilton@qut.edu.au

Received 18 March 2012; Accepted 2 May 2012

Academic Editors: R. Mamlok-Naaman, M. Martin, and M. Platsidou

Copyright ( $\odot 2012$ Lee-Ann M. Wilson et al. This is an open access article distributed under the Creative Commons Attribution License, which permits unrestricted use, distribution, and reproduction in any medium, provided the original work is properly cited.

It is suggested that all psychologists gain basic training in the types of complementary and alternative therapies (CAT) their clients may be using. As psychology students are the next cohort of health professionals who will inform future initiatives in the field, it is important to understand the factors which influence their decisions about CAT integration. Drawing on the theory of planned behavior, we investigated the beliefs that differentiate between psychology students who are high or low on willingness to access training in CAT for future practice use. Undergraduate psychology students $(N=106)$ completed a questionnaire assessing the likelihood of positive and negative consequences of accessing training and utilizing CAT within a psychological practice, important others' approval, and barriers preventing them from this integration behavior. Those students higher on willingness were more likely to endorse positive outcomes (e.g., offering a more holistic approach to therapy) of accessing CAT training for future practice use and to believe that important others (e.g., clients) would support this behavior. A regression analysis examining the relative importance of these belief sets broadly supported the belief-based analyses. These beliefs of student psychologists can inform educators and policy makers about CAT training and integration in psychology practice.

\section{Introduction}

The use of complementary and alternative therapies (CAT) has risen sharply over the past two decades, and this rise has not only impacted on the provision of medical care but also extends into psychological services. For example, in the United States, 64\% of psychotherapy clients reported using CAT [1]. Furthermore, between 1993 and 2000, there was a $120 \%$ increase in expenditure on alternative medicines and a $62 \%$ increase in expenditure on alternative therapists amongst the Australian general public [2]. Recent longitudinal evidence from South Australia indicates that this tendency is continuing [3]. This rise in the popularity and use of CAT suggests that many individuals with mental health disorders are seeking the advice of naturopaths or are combining yoga, meditation, or herbal therapies with traditional psychotherapy [4]. While significant research has been conducted exploring attitudes to CAT among medical professionals [5], there is an absence of similar, theory-based, investigations within the field of psychology.

1.1. CAT for Mental Health-Evidence and Attitudes. Within the health professions, there is an increased level of acceptance amongst practitioners for a number of CAT [6]. Furthermore, there is a growing body of evidence to support the use of some CAT for mental health issues. For example, St. John's wort, folate, vitamin B12, and amino acids, such as phenylalanine and S-adenosylmethionine (SAMe), have been reported to reduce symptoms of depression with few side effects [7]. Saeed et al. [8] reviewed randomised controlled trials of CAT treatments for depression and found 
that, in addition to the above nonprescription treatments, exercise and yoga can also be used to augment traditional psychotherapy. Similarly, Antonacci et al. [9] reviewed CAT treatments for anxiety disorders and found evidence to support the efficacy and use of exercise, yoga, kava, and the natural isomer of glucose, Intosol. Of course, where evidence in relation to efficacy is scarce, caution should be exercised. There is, however, a growing body of evidence to support the use of CAT as an adjunct to traditional psychotherapy.

Little is known about the ways in which psychologists are integrating CAT into psychological practice; however, the types of integration which have been reported by health professionals, including psychologists, fall into three broad categories. These include recommending CAT to clients, offering clients referrals to CAT practitioners, and accessing training in CAT therapies to integrate these therapies into practice [10]. In the United States, Bassman and Uellendahl [10] assessed psychologists' knowledge, attitudes, practices, and patterns of referral regarding CAT. Their findings indicated that most psychologists believed that CAT could aid in treatment. Respondents reported recommending CAT generally at relatively high rates and at significantly higher rates than they were offering specific referrals to CAT practitioners. Furthermore, a few of the psychologists surveyed reported that they were using CAT, including chiropractic, herbalism, acupuncture, and massage therapies, directly within their practice. White [11] reviewed the use of CAT for the treatment of psychological disorders and suggested that CAT integration could be efficacious for psychologists. White recommended that all psychologists should obtain basic training in regards to the types of CAT their clients may be using and that psychologists who wish to integrate should obtain more extensive training.

Although, it is useful to have an understanding of the factors which influence psychologists' choices about integrating CAT into therapy to inform both clinical practice policy and educational initiatives, it is useful also to examine the perspectives of psychology students regarding CAT. Psychology students are the next cohort of health professionals who will be in a position to direct future initiatives in the field. It may be helpful, then, to understand psychology students' perspectives about CAT by examining the underlying processes which guide their decision-making to determine any differences between those students who would or would not undertake CAT training for future professional use. One of the most commonly utilized decision-making models in the social and health domains is the theory of planned behavior (TPB; [12]).

1.2. Theory of Planned Behavior. According to the TPB, intention to perform a given behavior and the individual's actual control over performing the behavior predict behavioral performance. Intention, in turn, is influenced by the degree to which a person is in favor of performing a particular behavior (attitude), the degree to which a person feels social pressure to perform a behavior (subjective norms), and the degree to which a person feels they have control over performing the behavior (perceived behavioral control) [12]. Underlying the TPB is the assumption that the antecedents of attitude, subjective norms, and perceived behavioral control are corresponding salient beliefs, which reflect an individual's intention and subsequent behavior [12]. Attitudes are determined by the individual's beliefs about the likely outcomes of performing the behavior (behavioral beliefs) weighted by the positive or negative evaluations of these outcomes. Subjective norms relate to the individual's beliefs about important referents either approving or disapproving of a given behavior (normative beliefs) weighted by the individuals motivation to comply with these important individuals or groups. Perceived behavioral control is based on the individual's beliefs concerning the extent to which internal and external factors may inhibit or facilitate performance of a given behavior (control beliefs) weighted by the expected impact these factors would have on behavioral performance if they were present [12].

Given that it is suggested that the traditional evaluative items are not always essential for belief measurement [12], the current study only focused on the beliefs underlying students' perceptions about CAT training for future integration into psychology practice. Assessing the belief-based determinants of attitude, subjective norms, and perceived behavioral control allows researchers to establish the beliefs that differentiate those who perform a given behavior from those who do not. A number of studies have utilised the knowledge of these underlying beliefs to increase our understanding of health behavior in adults (e.g., [13]) and, in particular, CAT behavior [14, 15].

1.3. Willingness to Integrate CAT. In light of fact that psychology students have not yet experienced any clinical practice, it was decided that a measure of willingness to integrate CAT into psychological practice might be more appropriate than intention to do so, as willingness does not require participants to be aware of any workplace or professional constraints such as codes of conduct (i.e., they could indicate that given the opportunity they might be willing to integrate CAT into their future psychological practice rather than indicating an actual intention in regards to a possible future practice). Willingness has been included in TPB-based models previously (e.g., [16]), although it is most frequently examined in the context of the prototype willingness model (PWM) [17]. The PWM does not include perceived behavioral control but includes attitudes from the TPB and conceptualizes norms in terms of perceptions of what significant others actually do rather than what significant others think the person should do, as proposed by the TPB [17].

Wilson and White [15] assessed underlying beliefs of psychologists high and low on willingness or intentions to integrate CAT by either recommending CAT in general to their clients or referring their clients to specific CAT practitioners. Their findings indicated that beliefs underlying attitudes (e.g., being able to offer a more holistic approach to therapy), normative pressures from important others (e.g., clients), and perceptions of control (e.g., lack of knowledge about relevant CAT) discriminated between psychologists 
who were or were not willing to integrate CAT into their psychological practice. Interestingly, psychologists were much more willing to offer a general recommendation to CAT than to refer to CAT practitioners. Approximately $60 \%$ of psychologists indicated a favourable response (i.e., above the midpoint of the scale) for willingness to integrate by recommending CAT; however, only around 35\% indicated a willingness to integrate by offering referrals to specific CAT practitioners. Furthermore, in a recent qualitative study, Wilson and White [18] noted that psychology students, but not practicing psychologists, were relatively keen to integrate CAT by accessing training for integration into their future psychology practice.

1.4. The Current Study. Given that people's use of CAT has risen sharply over recent years [2], there is evidence to support the use of CAT as an adjunct to traditional psychotherapy $[8,9]$, and it is suggested that all psychologists should obtain basic training in the types of CAT their clients may be using [11], it is important to have an understanding of the underlying beliefs that guide CAT integration decision-making. Identifying the underlying beliefs that differentiate between those who would and would not be willing to access CAT training for integration into future clinical practice is important as it can help to inform clinical practice policy and educational initiatives aimed at encouraging such behaviors among psychologists. Furthermore, gaining this understanding from psychology students is especially important given that they are the next cohort of health professionals who will be in a position to direct future initiatives in the field. Using the TPB as a theoretical framework, this study aims to investigate beliefs that differentiate those psychology students who are willing to access CAT training for integration into their future psychology practice. Specifically, the study assessed psychology students' beliefs relating to accessing CAT training for future integration costs and benefits (behavioral beliefs), beliefs about important referents judgments of accessing CAT training for future integration (normative beliefs), and beliefs about inhibiting factors for accessing CAT training for future integration. Such belief-based analyses allow a distinction between subsamples that can assist in informing education and future policy directions in the important area of mental health.

\section{Method}

2.1. Participants. Students were invited by the first author at a core unit psychology lecture to participate in the study and were asked to complete the paper-based questionnaire. Participants $(N=106)$ were psychology undergraduate students from a major Australian university. The majority of the students were enrolled in first year psychology units $(89 \%)$ and indicated a preference of clinical $(42 \%)$ or counseling (32\%) psychology as their future area of professional interest. The study comprised of 77 females and
29 males and the mean age of participants was 24.55 years $(\mathrm{SD}=10.46$ years $)$.

2.2. Measures. The target behavior was assessing students' beliefs about integrating CAT into a future psychology practice by accessing training in a particular therapy and then integrating that therapy into their psychology practice. CAT was operationalised as a group of diverse medical and health care systems, practices, and products that are not presently considered to be part of conventional medicine.

This definition is consistent with the United States National Centre for Complementary and Alternative Medicines [19]. It is important to note that there are many and varied definitions of CAT. Many people do not include chiropractic, vitamins, massage, or meditation in their definition of CAT, although others do. The definition employed in this study was intentionally broad so that participants would think about those practices which they themselves consider to be complementary and/or alternative.

2.2.1. Elicitation Study. A pilot study with both practicing psychologists and psychology students was conducted $(N=$ 12: 6 psychologists and 6 students) to elicit the most commonly occurring behavioral, normative, and control beliefs as recommended by Fishbein and Ajzen [20]. Participants were interviewed individually and asked to describe the advantages and disadvantages of accessing CAT training for future integration into their psychology practice, any group or people who would approve or disapprove of them accessing CAT training for future integration into their psychology practice, and to discuss any factors or circumstances that might prevent and encourage them to access CAT training for future integration into their psychology practice. The most commonly reported advantages (e.g., being able to offer a more holistic approach to therapy) and disadvantages (e.g., comprising my professional practice), referents approving or disapproving (e.g., clients), and factors preventing accessing CAT training for future integration into psychology practice (e.g., lack of scientific evidence in support of CAT), reported by students, were used to assess the behavioral, normative, and control belief-based measures, respectively, in this study.

2.2.2. Main Questionnaire. All belief-based items were scored on 7-point Likert scales, scored extremely unlikely (1) to extremely likely (7). To assess behavioral beliefs, students were asked to rate how likely two costs and two benefits would result if they integrated CAT into a future psychology practice by accessing training in a particular therapy and then integrated that therapy into their psychology practice. For normative beliefs, students were asked to rate how likely four referents would think they should integrate CAT into a future psychology practice by accessing training in a particular therapy and then integrated that therapy into their psychology practice. Participants indicating how likely internal and external factors would prevent them from integrating CAT into a future psychology practice by accessing training in a particular therapy and then integrated that therapy into their psychology practice. For a full listing 
of belief-based items, see Table 1. The outcome measure of willingness was measured on a single item scale adopted from the prototype/willingness model [20]. Students were asked to respond to the question: "I would be willing (1), would not be willing (7) to integrate CAT into a future psychology practice by accessing training in a particular therapy and then integrating that therapy into my psychology practice."

2.3. Procedure. Prior to any data collection, the university human research ethics committee approved the study. Students were informed about the opportunity to participate in the study during one of their regularly scheduled classes. At the end of the class, students who were willing to participate were able to complete and return their survey before leaving. The student participants were offered a small number of credit points towards a research participation mark as a thank you gift for their involvement.

2.4. Statistics. For belief-based analyses, responses were recoded to indicate those who had low willingness (scores 3.1 to $7.0=1$ ) and high willingness (scores 1.0 to $3.0=2$ ) toward accessing CAT training for future integration into their psychology practice. Using SPSS 17.0, multivariate analyses of variance (MANOVA) were conducted to examine the influence of beliefs on the high- and low-willingness groups. To explore where the differences exist between the groups, dependent variables (i.e., beliefs) were examined at the univariate level. Bonferroni adjustments were used to control for familywise type 1 error.

A multiple regression analysis was conducted to assess the relative importance of the beliefs for predicting willingness to integrate CAT into a future psychology practice by accessing training in a particular therapy and then integrating that therapy into my psychology practice. The belief item score for each corresponding TPB belief-based composite variable was averaged to create the behavioral, normative, and control belief-based measures. No assumption is made that salient beliefs are internally consistent, and, as such, internal consistency is not a necessary feature of belief composites [12] and alpha coefficients were not computed. The dependent measure was based on a continuous scale reflecting willingness (as described above).

\section{Results}

3.1. Belief-Based Analyses. Seventy-two percent of participants indicated a favorable response (i.e., above the midpoint of the scale) for willingness to integrate CAT into a future psychology practice by accessing training in a particular therapy and then integrating that therapy into their psychology practice. Overall, Wilks' lambdas were significant for all three MANOVAs. According to Wilks' criterion, a significant multivariate effect was identified between the groups for behavioral beliefs, $F(4,99)=6.77, P<.001$, $\eta^{2}=.22$. Univariate analyses revealed that those students high on willingness were more likely than those students low on willingness to perceive both the positive outcomes of being able to offer a more holistic approach to therapy and of being able to avoid the use of medications where they are unnecessary or unwanted by the client.

A significant multivariate effect was also found between the groups for normative beliefs, $F(3,102)=4.49, P=.005$, $\eta^{2}=.12$. Univariate analyses indicated that those students high on willingness were more likely than those students low on willingness to report that clients, and complementary or alternative practitioners would think they should access training in CAT for future integration into their psychology practice.

Finally, a significant multivariate effect was identified between the groups for control beliefs, $F(3,102)=4.13$, $P=.008, \eta^{2}=.11$. Univariate analyses, however, revealed that there were no significant univariate differences between those students high and low on willingness. Students high and low on willingness were equally likely to perceive the barriers of lack of knowledge about relevant CATs, lack of scientific evidence in support of CAT, and absence of clear legal/professional bodies' guidelines as inhibiting their control over accessing CAT training for future integration into their psychology practice. Refer to Table 1 for the mean differences in the high and low willingness groups.

3.2. Analysis Predicting Willingness. The results of the multiple regression analysis are shown in Table 2. Together, the belief-based measures significantly predicted willingness, $F(3,102)=22.86, P<.001$, accounting for $40 \%$ of the variance of students' willingness to integrate CAT into a future psychology practice by accessing training in a particular therapy and then integrating that therapy into my psychology practice. Behavioral, normative beliefs, and control beliefs were significant predictors of student willingness.

\section{Discussion}

The current study, using a TPB belief-based approach, aimed to investigate whether belief-based differences exist between those students with high and low willingness to access CAT training for future integration into their psychology practice. The results revealed that the behavioral beliefs (cost and benefits) and normative beliefs (pressure to comply with important others approval or disapproval), but not any of the control beliefs (motivating or inhibiting factors), differed significantly. Additionally, the regression analysis revealed that the behavioral $(\beta=.40)$ and normative beliefs $(\beta=.31)$ were relatively more important than the control beliefs $(\beta=$ -.24) in predicting student willingness, although all three belief sets were revealed as significant predictors of students' willingness to access CAT training for future integration into their psychology practice.

4.1. Belief-Based Findings. For the behavioral beliefs, students who had high willingness to access CAT training for future integration into their practice were more likely than those low on willingness to believe that there would be positive outcomes of accessing CAT training for future practice use. Specifically, students high on willingness were more likely than those students low on willingness to believe 
TABLE 1: Mean differences in behavioral, normative, and control beliefs for students' willingness to access CAT training for future integration into their psychology practice.

\begin{tabular}{|c|c|c|}
\hline Willingness & Low $^{\mathrm{b}}$ willingness & High $^{\mathrm{b}}$ willingness \\
\hline Behavioural beliefs $^{\mathrm{a}}$ & $n=29$ & $n=75$ \\
\hline Being able to offer a more holistic approach to therapy & 4.38 & $5.52 * * *$ \\
\hline Clients feeling pressured to experience CAT & 3.90 & 3.37 \\
\hline $\begin{array}{l}\text { Being able to avoid the use of medications where they are } \\
\text { unnecessary or unwanted by the client }\end{array}$ & 4.38 & $5.25^{* * *}$ \\
\hline Compromising my professional reputation & 3.97 & 3.31 \\
\hline \multicolumn{3}{|l|}{$* * * P<.012^{\mathrm{a}}$} \\
\hline Normative beliefs & $n=30$ & $n=76$ \\
\hline Your clients & 4.13 & $4.89^{* * *}$ \\
\hline Complementary or alternative practitioners & 4.87 & $5.64^{* * *}$ \\
\hline Professional organisations & 3.47 & 4.07 \\
\hline \multicolumn{3}{|l|}{$* * * P<.016^{\mathrm{a}}$} \\
\hline Control beliefs & $n=30$ & $n=76$ \\
\hline Lack of knowledge about relevant CAT & 4.97 & 5.45 \\
\hline Lack of scientific evidence in support of CAT & 5.33 & 4.88 \\
\hline Absence of clear legal/professional bodies guidelines & 5.20 & 4.93 \\
\hline$* * * P<.016^{\mathrm{a}}$ & & \\
\hline
\end{tabular}

${ }^{a}$ Bonferroni adjustments used to control for familywise type 1 error.

${ }^{b}$ Low and high willingness groups were created based on a split at the scale midpoint. The different ratios of $n$ values between the intention groups across the three TPB belief-based categories reflect the presence of missing data.

TABLE 2: Regression analysis predicting willingness to access CAT training for future integration into their psychology practice.

\begin{tabular}{lllcr}
\hline Variable & $R$ & $R^{2}$ & $F$ & $d f$ \\
\hline Willingness & .63 & .40 & 22.86 & 3,102 \\
Behavioral beliefs & & & & $.40^{* * *}$ \\
Normative beliefs & & & & $.31^{* *}$ \\
Control beliefs & & & $-.24^{* *}$ \\
\hline$* *$ r
\end{tabular}

**P $\leq .01, * * * P<.001$.

that accessing CAT training for future integration into their practice would enable them to offer a more holistic approach to therapy and to avoid the use of medications where they are unnecessary or unwanted by the client. This finding suggests that students are considering what might be in the best interests of the client and any attempt to influence future behavior could focus on the specific advantages and disadvantages for client wellbeing and mental health outcomes. These results broadly support the findings of previous studies examining attitudinal beliefs underlying CAT willingness $[14,15]$; specifically, practicing psychologists who are more willing to integrate CAT believe also that it provides a more holistic approach to therapy [15].

For the normative beliefs, students who had high willingness to access CAT training for future integration into their practice were more likely than those low on willingness to believe that those people/groups important to them (viz., their clients and complementary or alternative practitioners) would think they should access CAT training for future practice use. These findings are consistent with previous research in the CAT domain in that practicing psychologists who are more willing to integrate CAT believe also that their clients and complementary or alternative practitioners would approve of the behavior [15]. Therefore, should any parties wish to increase students' willingness to undertake CAT training for future integration into their psychology practice, a focus should be on emphasizing strategies that explicitly show these important others approving of students accessing CAT training for future practice use.

Finally, for the control beliefs, there was no difference between willingness groups with regards to inhibiting factors. This finding indicates that those students high and low on willingness perceive equally the control factors of lack of knowledge about CAT, lack of scientific evidence in support of CAT, and the absence of clear legal/professional bodies' guidelines, as inhibiting their control over accessing CAT training for future practice use. It is possible, given the student sample, that the potential barriers of accessing CAT training for future integration into practice are not yet fully understood by this group and, therefore, all students perceive these barriers as important to future professional practice. This explanation is somewhat supported by previous research which found practicing psychologists who are more willing to integrate CAT, compared to those less 
willing, perceive these factors (i.e., lack of knowledge about CATs, lack of scientific evidence in support of CAT, absence of clear legal/professional bodies guidelines) as less likely to inhibit their control over recommending CAT to their clients [15]. Based on these findings, then, highlighting the likely barriers of accessing CAT training for future practice use seems unlikely to persuade students to undertake CAT training for future integration into psychology practice.

4.2. Predicting Willingness. The results of the regression analysis broadly supported the belief-based analyses in that behavioral $(\beta=.40)$ and normative beliefs $(\beta=.31)$ were revealed as relatively more important than the control beliefs $(\beta=-.24)$ in predicting student willingness, although all three belief sets were significant accounting for $40 \%$ of the variance. These findings suggest that the more students perceive that there are benefits associated with accessing CAT training for future integration into their psychology practice the more they think that important referents approve of their accessing CAT training for future integration use, and the less likely control factors were perceived as inhibiting undertaking this behavior the more students were willing to access CAT training for future integration into their psychology practice. In light of these findings, policy and educational initiatives aimed at promoting students' willingness toward accessing CAT training for future use in psychology practice should place emphasis on highlighting the behavioral and normative beliefs. In addition, given that control beliefs also predicted student willingness, future research may benefit from a continued exploration of the underlying inhibiting and motivating factors guiding students' decision-making toward this behavior.

Interestingly, $72 \%$ of psychology students reported a favorable response for their willingness to integrate CAT into a future practice by accessing training and then using CAT within their practice. This favorable response is in contrast to earlier findings by Wilson and White [15] that psychologists were much less likely to be willing to integrate by offering recommendations or referrals. While the behaviors are different, other studies, including Bassman and Uellendahl [10] and Wilson and White [18], have found that psychologists are much more willing to integrate through recommending or referral than they are to access training and incorporate CAT into their practice. This apparent difference in perspective between students and practicing psychologists may reflect changing perspectives within the discipline over time or may say something about the constraints realized only when practicing as a psychologist. Either way, this difference in perspectives should be investigated in further research.

4.3. Strengths and Limitations. This research has a number of strengths including the identification of underlying beliefs in a unique sample (i.e., psychology students) for an important integration topic. Second, this study explores an issue which is of growing concern among practitioners and their governing bodies as well as across disciplines and within the general public. There is a mandate within the health disciplines to provide the best care available and to treat the "whole person," and yet there are a number of limitations to a practitioner's capacity to do this. This study shows that psychologists in training take this mandate seriously and are keen to consider integrative treatment options.

The current study also has a number of limitations. First, the sample size was relatively small; however, the majority of students offered the chance to participate did so, and, as the course they were attending was a required unit, it may be assumed that the participants were representative of psychology students in general. Second, the sample contained a greater number of female students. Although this imbalance reflects both the higher proportion of females studying undergraduate psychology and the proportion of males and females actually practicing psychology in Australia, it would be worthwhile to explore gender differences with a larger sample as it is possible that male students may have different beliefs about CAT training and integration, at least as can be inferred from reported levels of personal use [3]. Finally, the broad definition of CAT used in the current study may limit the generalisability of the findings when referring to specific therapies. The underlying beliefs for subcategories of CAT might show some differences to the beliefs identified in this study. For example, students underlying beliefs regarding alternative therapies that have greater scientific evidence to support their usage (e.g., yoga, exercise, St. John's wort, folate, vitamin B12, and amino acids such as phenylalanine and S-adenosylmethionine (SAMe); [7-9]) might be different for beliefs held toward alternative therapies that are less supported by the literature or toward therapies that may be illsuited to the mode of CAT integration explored in this study, such as touch therapies.

4.4. Implications of the Research. Overall, the findings from the current study indicate that student psychologists consider their own attitudinal views as well as the perceived approval from those who are important to them, in forming their decisions to be willing to access CAT training for future integration into psychology practice. Specifically, the findings suggest that attention to benefits and considering the social approval of important others in understanding CAT decision-making may influence students' willingness to access CAT training for future practice use.

To improve students' willingness to access CAT training for future integration, therefore, educational authorities should highlight the benefits of CAT for clients wellbeing and mental health outcomes, specifically emphasizing that accessing CAT training for future practice use will allow them, as a health practitioner, to offer their clients a more holistic approach to therapy and avoid the use of medications where they are unnecessary or unwanted by the client. In addition, having clients and complementary or alternative practitioners approve of psychology students accessing CAT training for future practice use may be useful to increase student willingness. However, it may not be beneficial for educators and policymakers in trying to encourage students to undertake CAT training for future integration into psychology practice to focus on the likely barriers of doing so, although further research is needed here given that 
control beliefs predicted willingness. Given the increasing numbers of people using CAT for mental health disorders, this research represents the beginning of what is likely to be an ongoing investigation into the motives and directions for CAT training and integration within the field of psychology.

\section{References}

[1] G. Elkins, J. Marcus, M. H. Rajab, and S. Durgam, "Complementary and alternative therapy use by psychotherapy clients," Psychotherapy, vol. 42, no. 2, pp. 232-235, 2005.

[2] A. H. MacLennan, D. H. Wilson, and A. W. Taylor, "The escalating cost and prevalence of alternative medicine," Preventive Medicine, vol. 35, no. 2, pp. 166-173, 2002.

[3] A. H. MacLennan, S. P. Myers, and A. W. Taylor, "The continuing use of complementary and alternative medicine in South Australia: costs and beliefs in 2004," Medical Journal of Australia, vol. 184, no. 1, pp. 27-31, 2006.

[4] A. C. Page, R. Jones, and F. Wilson, "Survey of West Australian anxiety support group participants' views on treatment processes and outomes," Australian Psychologist, vol. 39, no. 3, pp. 208-211, 2004.

[5] L. L. Halcón, L. L. Chlan, M. J. Kreitzer, and B. J. Leonard, "Complementary therapies and healing practices: faculty/ student beliefs and attitudes and the implications for nursing education," Journal of Professional Nursing, vol. 19, no. 6, pp. 387-397, 2003.

[6] M. V. Pirotta, M. M. Cohen, V. Kotsirilos, and S. J. Farish, "Complementary therapies: have they become accepted in general practice?" Medical Journal of Australia, vol. 172, no. 3, pp. 105-109, 2000.

[7] A. F. Jorm, H. Christensen, K. M. Griffiths, and B. Rodgers, "Effectiveness of complementary and self-help treatments for depression," Medical Journal of Australia, vol. 176, supplement 10, pp. S84-S96, 2002.

[8] S. A. Saeed, R. M. Bloch, D. J. Antonacci, C. E. Davis III, and C. Manuel, "CAM for your depressed patient: 6 recommended options," Current Psychology Online, vol. 8, no. 10, 2009.

[9] D. J. Antonacci, R. M. Bloch, C. E. Davis III, C. Manuel, and S. A. Saeed, "CAM for your anxious patient: what the evidence says," Current Psychology Online, vol. 9, no. 10, 2010.

[10] L. E. Bassman and G. Uellendahl, "Complementary/alternative medicine: ethical, professional, and practical challenges for psychologists," Professional Psychology, vol. 34, no. 3, pp. 264-270, 2003.

[11] K. P. White, "What psychologists should know about homeopathy, nutrition, and botanical medicine," Professional Psychology, vol. 40, no. 6, pp. 633-640, 2009.

[12] I. Ajzen, "The theory of planned behavior," Organizational Behavior and Human Decision Processes, vol. 50, no. 2, pp. 179211, 1991.

[13] K. M. White, D. J. Terry, C. Troup, and L. A. Rempel, "Behavioral, normative and control beliefs underlying lowfat dietary and regular physical activity behaviors for adults diagnosed with type 2 diabetes and/or cardiovascular disease," Psychology, Health and Medicine, vol. 12, no. 4, pp. 485-494, 2007.

[14] E. L. O'Connor and K. M. White, "Intentions and willingness to use complementary and alternative medicines: what potential patients believe about CAMs," Complementary Therapies in Clinical Practice, vol. 15, no. 3, pp. 136-140, 2009.

[15] L. M. Wilson and K. M. White, "Identifying the attitudinal, normative, and control beliefs underlying psychologists' willingness to integrate complementary and alternative therapies into psychological practice," in Proceedings of the 43rd APS Annual Conference, In Psychology Leading Change 2008, Hobart, Australia, September 2008.

[16] M. K. Hyde and K. M. White, "Are organ donation communication decisions reasoned or reactive? A test of the utility of an augmented theory of planned behaviour with the prototype/willingness model," British Journal of Health Psychology, vol. 15, no. 2, pp. 435-452, 2010.

[17] F. X. Gibbons, M. Gerrard, J. Ouelette, and B. Burzette, "Discrimininating between behavioral intention and behavioral willingness: cognitive antecedents to adolescent health risk," in Understanding and Changing Health Behaviour: From Health Beliefs to Self-Regulation, P. Norman and C. Abraham, Eds., Harwook Academic publishers, Amsterdam, The Netherlands, 2000.

[18] L.-A. M. Wilson and K. M. White, "Integrating complementary and alternative therapies into psychological practice: a qualitative analysis," Australian Journal of Psychology, vol. 63, no. 4, pp. 232-242, 2011.

[19] NCCAM, What is Complementary and Alternative Medicine (CAM)?, http://www.nccam.nih.gov/health/whatiscam/index .htm, 2006.

[20] M. Fishbein and I. Ajzen, Belief, Attitude, Intention, and Behavior: An Introduction to Theory and Research, AddisonWesley, Reading, Mass, USA, 1975. 

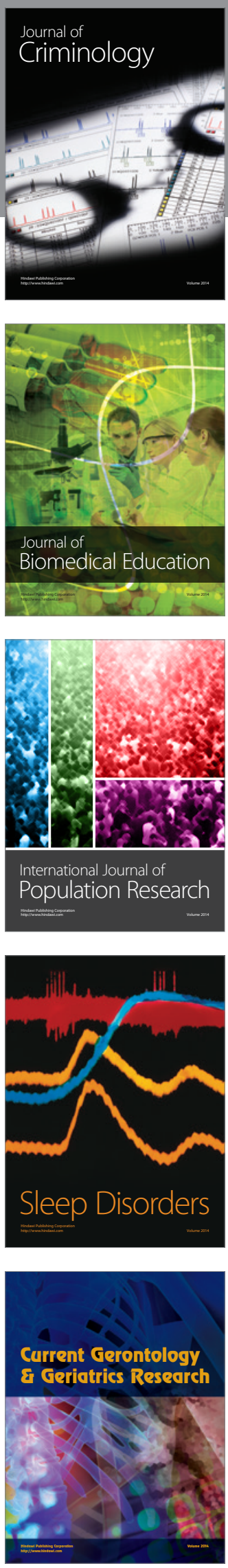
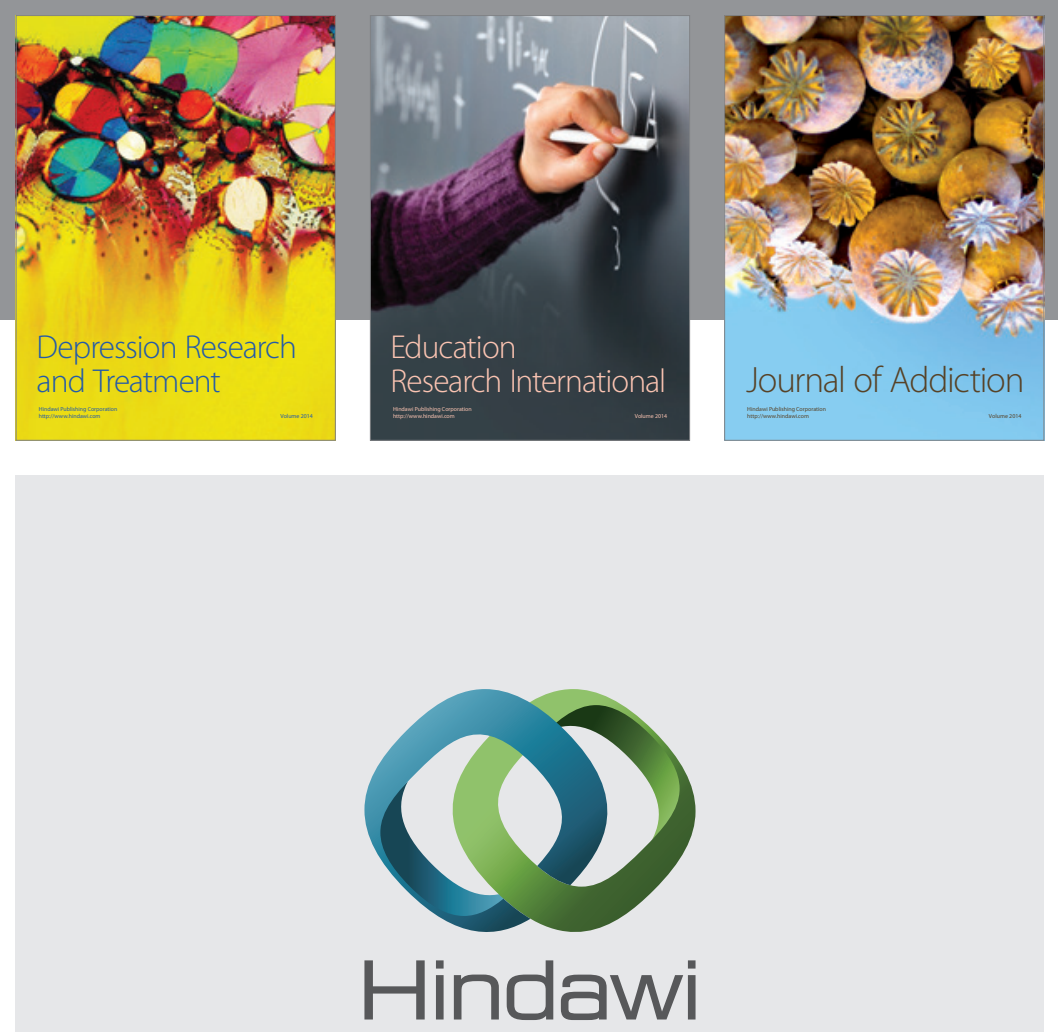

Submit your manuscripts at

http://www.hindawi.com

Child Development Research
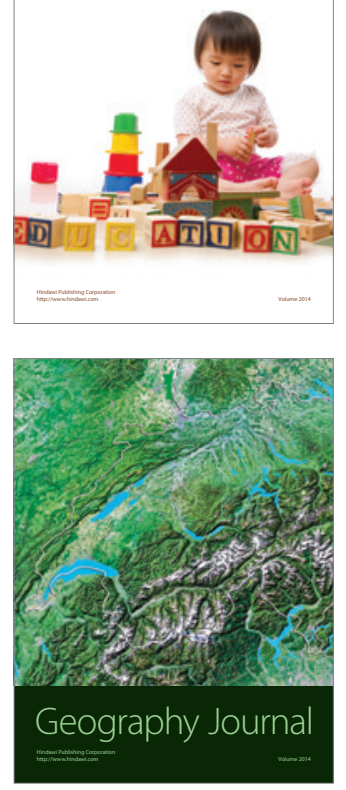

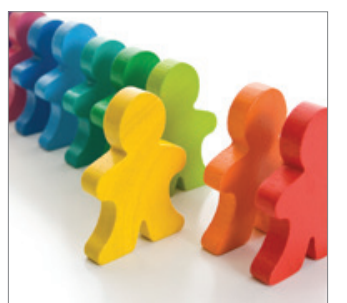

Autism

Research and Treatment
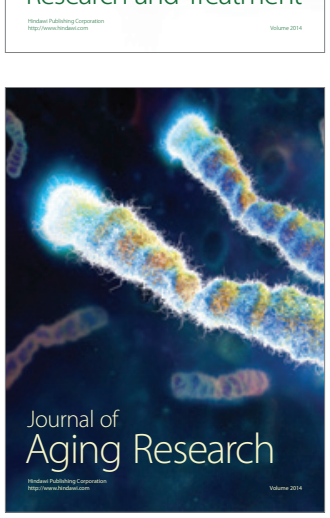
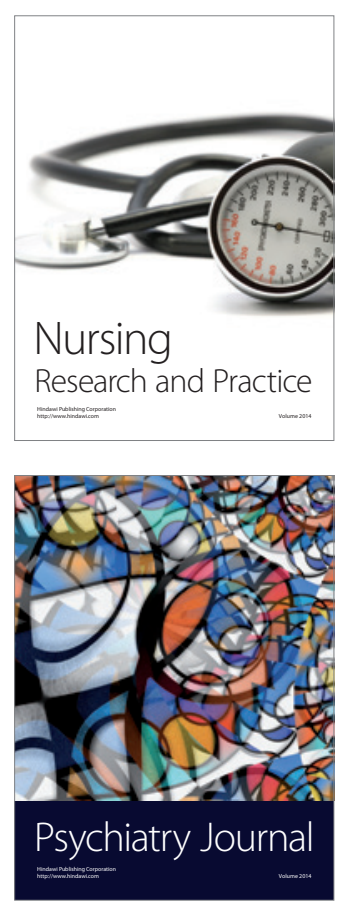
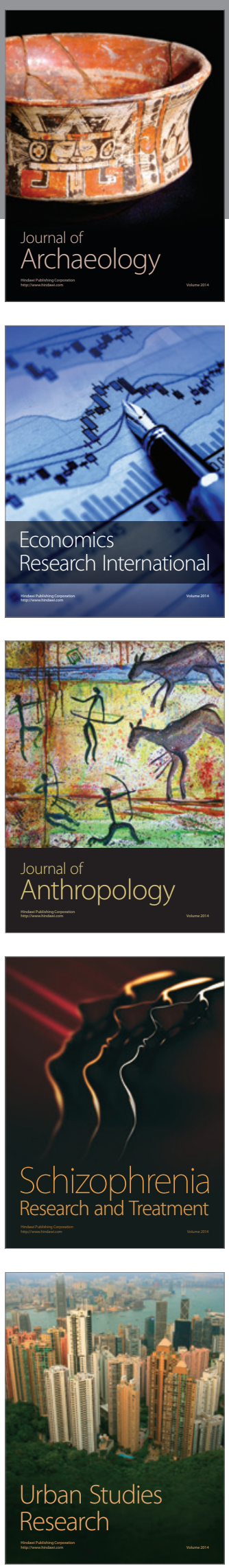